\title{
STRATEGY-PROOF MECHANISM DESIGN WITH NON-QUASILINEAR PREFERENCES: EX-POST REVENUE MAXIMIZATION FOR AN ARBITRARY NUMBER OF OBJECTS
}

\author{
Ryosuke Sakai \\ Shigehiro Serizawa
}

October 2020

The Institute of Social and Economic Research

Osaka University

6-1 Mihogaoka, Ibaraki, Osaka 567-0047, Japan 


\title{
Strategy-proof mechanism design with non-quasilinear preferences: Ex-post revenue maximization for an arbitrary number of objects*
}

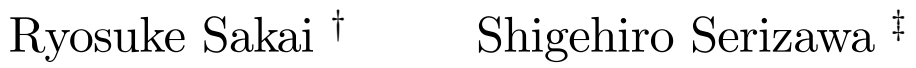

October 25, 2020

\begin{abstract}
We consider the multi-object allocation problem with monetary transfers where each agent obtains at most one object (unit-demand). We focus on allocation rules satisfying individual rationality, non-wastefulness, equal treatment of equals, and strategy-proofness. Extending the result of Kazumura et al. (2020B), we show that for an arbitrary number of agents and objects, the minimum price Walrasian is the unique ex-post revenue maximizing rule among the rules satisfying no subsidy in addition to the four properties, and that no subsidy in this result can be replaced by no bankruptcy on the positive income effect domain.
\end{abstract}

JEL classification: D82, D47, D63.

Keywords: Multi-object allocation problem, Strategy-proofness, Ex-post revenue maximization, Minimum price Walrasian rule, Non-quasi-linear preference, Equal treatment of equals, Non-wastefulness.

\footnotetext{
${ }^{*}$ We gratefully acknowledge financial support from the Joint Usage/Research Center at ISER, Osaka University, Osaka University's International Joint Research Promotion Program (Type A), and the Japan Society for the Promotion of Science (15H05728, 19J10221, 20H05631)

${ }^{\dagger}$ Graduate School of Economics, Osaka University. Email: tge012sr@student.econ.osaka-u.ac.jp

${ }^{\ddagger}$ Institute of Social and Economic Research, Osaka University. Email: serizawa@iser.osaka-u.ac.jp
} 


\section{Introduction}

This is to extend Kazumura et al. (2020B) to a general case of an arbitrary number of agents and objects. They consider the multi-object allocation problem with monetary transfers where each agent obtains at most one object (unit-demand). A (consumption) bundle is a pair of object and payment. Each agent has a continuous preference relation over bundles satisfying the possibility of compensation, money monotonicity, and object desirability. Such preferences are called classical. The classical domain is the class of all classical preferences.

An (allocation) rule, or simply rule chooses, for each preference profile, the object each agent receives and how much each agent pays. We focus on the following four properties of rules. A rule is desirable if it satisfies those properties.

(i) Individual rationality requires that each agent's bundle is at least as good as receiving nothing with no payment. Without this condition, agents does not participate the rule voluntarily. (ii) Non-wastefulness means that no agent prefers his own bundle to unassigned object with no payment. This condition is the property of weak efficiency. (iii) Equal treatment of equals requires that if there are two agents with same preferences, then their bundles are indifference for their preferences. This is the weak condition of fairness. (iv) Strategy-proofness is the incentive compatible condition, which means that no agent has incentive to misreport his preference.

In multi-object allocation problem, for each preference profile, Walrasian equilibrium exists (Alkan and Gale, 1990), and Demange and Gale (1985) show that the set of Walrasian prices has a lattice structure; that is, there is the minimum price Walrasian equilibrium for each preference profile. The minimum price Walrasian rule is desirable (Demange and Gale, 1985), and it satisfies efficiency and no subsidy (no-negative monetary transfer).

A subdomain of the classical domain is rich if for each object, there is a preference in the subdomain which demands only the object for some price and demands no object for some other price. ${ }^{1}$ Kazumura et al. (2020B) illustrate that various domains including the quasi-linear domain are rich, and show that in the case where the number of agents is greater than the number of objects, the minimum price Walrasian rule is the unique ex-post revenue maximizing rule among the desirable rules satisfying no subsidy on a rich domain. We extend their result to a general case of an arbitrary number of agents and objects.

This article is organized as follows. Section 2 introduces the model and basic concepts. Section 3 checks the properties of minimum price Walrasian rules. Our results are in Section 4. Section 5 provides proofs. Section 6 concludes.

\footnotetext{
${ }^{1}$ See Subsection 4.1 for the formal definition of richness.
} 


\section{The model}

Let $N=\{1,2, \cdots, n\}$ be the set of agents and $M=\{1,2, \cdots, m\}$ be the set of different objects. Not consuming an object in $M$ is called consuming the "null object". Let $L \equiv M \cup\{0\}$, where 0 denotes the null object. Each agent consumes at most one object. A typical (consumption) bundle for agent $i$ is a pair $z_{i}=\left(x_{i}, t_{i}\right) \in L \times \mathbb{R}$ : agent $i$ receives object $x_{i}$ and pays $t_{i}$.

Each agent has a complete and transitive preference relation $R_{i}$ over $L \times \mathbb{R}$. Let $I_{i}$ and $P_{i}$ be the indifference relation and strict preference relation associated with $R_{i}$. A typical class of preferences is denoted by $\mathcal{R}$. We call $\mathcal{R}^{n}$ a domain. $\mathcal{R}$ is classical if it satisfies the following assumptions:

1. Continuity: For each $z_{i} \in L \times \mathbb{R}$, the sets $\left\{z_{i}^{\prime} \in L \times \mathbb{R}: z_{i}^{\prime} R_{i} z_{i}\right\}$ and $\left\{z_{i}^{\prime} \in L \times \mathbb{R}\right.$ : $\left.z_{i} R_{i} z_{i}^{\prime}\right\}$ are closed.

2. Possibility of compensation: For each pair $a, b \in L$ and each $t \in \mathbb{R}$, there exist $t^{\prime}, t^{\prime \prime} \in \mathbb{R}$ such that $(a, t) R_{i}\left(b, t^{\prime}\right)$ and $\left(b, t^{\prime}\right) R_{i}\left(a, t^{\prime \prime}\right)$.

3. Money monotonicity: For each $a \in L$ and each pair $t, t^{\prime} \in \mathbb{R}$, if $t<t^{\prime}$, then $(a, t) P_{i}\left(a, t^{\prime}\right)$.

4. Object desirability: For each $a \in M$ and each $t \in \mathbb{R},(a, t) P_{i}(0, t)$.

Let $\mathcal{R}^{C}$ be the set of classical preferences. We assume that $\mathcal{R} \subseteq \mathcal{R}^{C}$. A preference profile is a list of preferences $R \equiv\left(R_{1}, \cdots, R_{n}\right)$. Given $i \in N$ and $N^{\prime} \subseteq N$, let $R_{-i} \equiv$ $\left(R_{j}\right)_{j \neq i}$ and $R_{-N^{\prime}} \equiv\left(R_{j}\right)_{j \in N \backslash N^{\prime}}$.

A (feasible) object allocation is an $n$-tuple $x=\left(x_{1}, x_{2}, \ldots, x_{n}\right) \in L^{n}$ such that for each pair $i, j \in N$, if $x_{i}=x_{j}$, then $x_{i}=x_{j}=0$. Let $A$ be the set of all object allocations. An allocation is a pair of an object allocation and a vector of payments, $z=\left(\left(x_{1}, x_{2}, \ldots, x_{n}\right),\left(t_{1}, t_{2}, \ldots, t_{n}\right)\right) \in A \times \mathbb{R}^{n}$. Given $z \in A \times \mathbb{R}^{n}$ and $i \in N, z_{i}=$ $\left(x_{i}, t_{i}\right) \in L \times \mathbb{Z}$ denotes the bundle of agent $i$.

An (allocation) rule associates an allocation to each preference profile. Formally, a rule is a mapping $f=(x, t): \mathcal{R}^{n} \rightarrow A \times \mathbb{R}^{n}$. Given a rule $f$ and a preference profile $R \in \mathcal{R}^{n}$, agent $i$ 's assignment under $f$ at $R$ is denoted by $f_{i}(R)$. Moreover, we write $f_{i}(R) \equiv\left(x_{i}(R), t_{i}(R)\right) \in L \times \mathbb{R}$, where $x_{i}(R)$ denotes $i$ 's object assignment and $t_{i}(R)$ denotes his payment. We define $f(R) \equiv\left(f_{1}(R), \cdots, f_{n}(R)\right)$.

Definition 1. An allocation rule $f=(x, t)$ is desirable if it satisfies the following axioms:

- Individual rationality: For each $R \in \mathcal{R}^{n}$ and each $i \in N, f_{i}(R) R_{i}(0,0)$.

- Non-wastefulness: For each $R \in \mathcal{R}$, each $i \in N$ and each $a \in M$, if $x_{i}(R) \neq a$ and $(a, 0) P_{i} f_{i}(R)$, then there is $j \neq i$ such that $x_{j}(R)=a$. 
- Equal treatment of equals: For each $R \in \mathcal{R}^{n}$ and each $i, j \in N$ with $R_{i}=R_{j}$, $f_{i}(R) I_{i} f_{j}(R)$.

- Strategy-proofness: For each $R \in \mathcal{R}^{n}$, each $i \in N$ and each $R_{i}^{\prime} \in \mathcal{R}, f_{i}(R) R_{i} f_{i}\left(R_{i}^{\prime}, R_{-i}\right)$.

Non-wastefulness means that no agent prefers unassigned object with no payment to his own bundle. This condition is a weak condition of efficiency.

We say the allocation rule satisfies no-wastage if for each $R \in \mathcal{R}^{n}$ and each $a \in M$, there is $i \in N$ such that $x_{i}(R)=a$. Note that when $n<m$, no rule satisfies no-wastage. Kazumura et al. (2020B) define desirability by (i) individual rationality, (ii) no-wastage, (iii) equal treatment of equals, and (iv) strategy-proofness, which is different from our definition. However, since they assume that the number of agents is greater than the number of objects, no-wastage implies no-wastefulness. Thus, it is natural to relax nowastage to non-wastefulness.

Remark 1. (i) If $n<m$, no rule satisfies no-wastage. (ii) If $n \geq m$ and $f$ satisfies no-wastage, then it is non-wasteful.

Definition 2. An allocation rule $f=(x, t)$ satisfies no subsidy if for each $R \in \mathcal{R}^{n}$ and each $i \in N, t_{i}(R) \geq 0$.

Let $p=\left(p_{1}, p_{2}, \cdots, p_{m}\right) \in \mathbb{R}_{+}^{m}$ be a price vector. We assume that the price of null object is equal to zero; that is, $p_{0}=0$. Given $i \in N, R_{i} \in \mathcal{R}$, and $p \in \mathbb{R}_{+}^{m}$, let $D\left(R_{i}, p\right) \equiv\left\{a \in L: \forall b \in L,\left(a, p_{a}\right) I_{i}\left(b, p_{b}\right)\right\}$ denote the demand set of agent $i$ with $R_{i}$ at $p$.

Next, we define the concept of Walrasian equilibrium. It is a pair of a price vector and an allocation such that each agent an object he demands and pays its price, and the price of an unassigned object is zero.

Definition 3. Given $R \in \mathcal{R}^{n}$, a pair $((x, t), p) \in\left(A \times \mathbb{R}^{n}\right) \times \mathbb{R}_{+}^{m}$ is a Walrasian equilibrium for $R$ if

WE-i: for each $i \in N, x_{i} \in D\left(R_{i}, p\right)$ and $t_{i}=p_{x_{i}}$; and

WE-ii: for each $a \in M \backslash\left\{x_{j}\right\}_{j \in N}, p_{a}=0$.

Given $R \in \mathcal{R}^{n}$, let $W(R)$ be the set of Walrasian equilibria for $R$, and define

$$
Z(R) \equiv\left\{z \in A \times \mathbb{R}^{n}: \exists p \in \mathbb{R}_{+}^{m} \text { s.t. }(z, p) \in W(R)\right\}
$$

and

$$
P(R) \equiv\left\{p \in \mathbb{R}_{+}^{m}: \exists z \in A \times \mathbb{R}^{n} \text { s.t. }(z, p) \in W(R)\right\}
$$




\section{Facts}

Fact 1 (Alkan and Gale, 1990). For each $R \in \mathcal{R}^{n}$, there is a Walrasian equilibrium; that is, $W(R) \neq \emptyset$.

Fact 2 (Demange and Gale, 1985). For each $R \in \mathcal{R}^{n}$, there is $p \in \mathbb{R}_{+}^{m}$ such that for each $p^{\prime} \in P(R), p \leq p^{\prime} .^{2}$

Given $R \in \mathcal{R}^{n}$, we denote the minimum Walrasian price for $R$ by $p^{\min }(R)$ and define

$$
Z^{\min }(R) \equiv\left\{z \in A \times \mathbb{R}^{n}:\left(z, p^{\min }(R)\right) \in W(R)\right\} .
$$

We say an allocation rule $f$ is a minimum price Walrasian rule if for each $R \in \mathcal{R}^{n}$, $f(R) \in Z^{\min }(R)$.

Fact 3 (Demange and Gale, 1985). The minimum price Walrasian rule $f$ is strategyproof. $^{3}$

By the definition of Walrasian equilibrium, the minimum price Walrasian rule satisfies individual rationality, non-wastefulness, and equal treatment for equals, and so it is desirable; moreover, it satisfies no subsidy.

Fact 4 (Demange and Gale, 1985). The minimum price Walrasian rule $f$ is desirable and satisfies no subsidy.

\section{Results}

Definition 4. A rule $f=(x, t)$ revenue dominates $g=\left(x^{\prime}, t^{\prime}\right)$ such that for each $R \in \mathcal{R}^{n}, \sum_{i \in N} t_{i}(R) \geq \sum_{i \in N} t_{i}^{\prime}(R)$.

A rule is ex-post revenue optimal among a class of rules if it is in a class of rule and revenue dominates each rules in the class. We explore ex-post revenue optimal rules among the class of desirable satisfying no subsidy on rich domains in Subsection 4.1, and among the class of desirable satisfying no bankruptcy on the positive income effect domain in Subsection 4.2.

\footnotetext{
${ }^{2} p \leq p^{\prime}$ means that $p_{a} \leq p_{a}^{\prime}$ for each $a \in M$.

${ }^{3}$ Precisely, they show that the minimum price Walrasian rule is group strategy-proofness; we say a rule $f$ is group strategy-proof if for each $R \in \mathcal{R}^{n}$ and each $N^{\prime} \subseteq N$, there is no $R_{N^{\prime}}^{\prime} \in \mathcal{R}^{\left|N^{\prime}\right|}$ such that for each $i \in N, f_{i}\left(R_{N^{\prime}}^{\prime}, R_{-N^{\prime}}\right) P_{i} f_{i}(R)$.
} 


\subsection{The result on Rich domains}

We define the richness of a domain.

Definition 5. A domain $\mathcal{R}$ is rich if for each $a \in M$ and each $p \in \mathbb{R}_{+}^{m}$ with $p_{a}>0$ and $p_{b}=0$ for each $b \in M \backslash\{a\}$ and for each $p^{\prime}>p,{ }^{4}$ there is $R_{i} \in \mathcal{R}$ such that

$$
D\left(R_{i}, p\right)=\{a\} \text { and } D\left(R_{i}, p^{\prime}\right)=\{0\} .
$$

Fact 5 (Theorem 1 in Kazumura et al., 2020B). Let $\mathcal{R}$ be rich and $n>m$. The minimum price Walrasian rule is the unique ex-post revenue optimal rule among the class of desirable rules satisfying no subsidy.

Fact 6 (Morimoto and Serizawa, 2015). Let $R \in \mathcal{R}^{n}$ and $p=p^{\min }(R)$. Then, (i) if $n>m$, then for each $a \in M, p_{a}>0$, and (ii) if $n \leq m$, then there is $a \in M$ such that $p_{a}=0$.

In Kazumura et al. (2020B), the proof of Fact 5 depends on Fact 6 (i). However, when the number of agents is less or equal to the number of objects, we cannot use their method directly.

Theorem 1 shows that for an arbitrary number of agents and objects, the minimum price Walrasian rule is revenue optimal among the same class on a rich domain.

Theorem 1. Let $\mathcal{R}$ be rich. The minimum price Walrasian rule is the unique ex-post revenue optimal rule among the class of desirable rules satisfying no subsidy.

\subsection{The result on the positive income effect domain}

We define the positive income effect domain.

Definition 6. A preference $R_{i}$ satisfies positive income effect if for each $a, b \in L$ and each $t, t^{\prime} \in \mathbb{R}$ with $t<t^{\prime}$ and $\left(b, t^{\prime}\right) I_{i}(a, t)$, for each $\delta>0$,

$$
\left(b, t^{\prime}-\delta\right) P_{i}(a, t-\delta) .
$$

Let $\mathcal{R}^{++}$be the set of positive income effect preferences.

Definition 7. A rule $f$ satisfies no bankruptcy if there is $l \leq 0$ such that for each $R \in \mathcal{R}^{n}, \sum_{i \in N} t_{i}(R) \geq l$.

Fact 7 (Kazumura et al., 2020B). Let $\mathcal{R} \supseteq \mathcal{R}^{++}$and $n>m$. The minimum price Walrasian rule is the unique ex-post revenue optimal rule among the class of desirable rules satisfying no bankruptcy.

Similarly to Fact 7, but for an arbitrary number of agents and objects, we have:

\footnotetext{
${ }^{4} p^{\prime}>p$ means that $p_{a}^{\prime}>p_{a}$ for each $a \in M$.
} 
Theorem 2. Let $\mathcal{R} \supseteq \mathcal{R}^{++}$. The minimum price Walrasian rule is the unique ex-post revenue optimal rule among the class of desirable rules satisfying no bankruptcy.

Since the classical domain is a rich domain including the positive income effect domain, we have Corollary 1:

Corollary 1. The minimum price Walrasian rule is the unique ex-post revenue optimal rule among the class of desirable rules satisfying no bankruptcy on the classical domain.

\subsection{Efficiency}

Finally, we discuss the property of efficiency.

Definition 8. A rule $f=(x, t)$ satisfies efficiency if for each $R \in \mathcal{R}^{n}$, there is no allocation $z \in A \times \mathbb{R}^{n}$ such that (i) for each $i \in N, z_{i} R_{i} f_{i}(R)$, (ii) for some $j \in N$, $z_{j} P_{j} f_{j}(R)$ and (iii) $\sum_{k \in N} t_{k} \geq \sum_{k \in N} t_{k}(R)$.

Since ex-post revenue optimal rule is the minimum price Walrasian rule and it is an efficient rule (Morimoto and Serizawa, 2015), ex-post revenue optimal rule is efficient (Kazumura et al., 2020B). By the results of Kazumura et al. (2020B) and ours, we have:

Corollary 2. Let $\mathcal{R}$ be rich. For each $n, m \in \mathbb{N}$, if $f$ is ex-post revenue optimal among desirable rules satisfying no subsidy, then $f$ is efficient.

Corollary 3. Let $\mathcal{R} \supseteq \mathcal{R}^{++}$. For each $n, m \in \mathbb{N}$, if $f$ is ex-post revenue optimal among desirable rules satisfying no bankruptcy, then $f$ is efficient.

\section{Proofs}

Given $i \in N, R_{i} \in \mathcal{R}, a \in L$ and $(b, t) \in L \times \mathbb{R}_{+}$, the compensated valuation $V^{R_{i}}(a ;(b, t))$ of $a$ from $(b, t)$ for $R_{i}$ is the value such that $\left(a, V^{R_{i}}(a ;(b, t))\right) I_{i}(b, t)$.

Fact 8. Let $f$ satisfies individual rationality. Let $R \in \mathcal{R}^{n}, i \in N$ and $z \in Z(R)$. If $z_{i} P_{i} f_{i}(R)$, then $x_{i} \neq 0$.

Fact 9 (Lemma 1 in Kazumura et al., 2020B). Let $g=\left(x^{\prime}, t^{\prime}\right)$ be a minimum price Walrasian rule. Then, for each rule $f$ and each $R \in \mathcal{R}^{n}$,

$$
\left[\forall i \in N, f_{i}(R) R_{i} g_{i}(R)\right] \Rightarrow \sum_{i \in N} t_{i}^{\prime}(R) \geq \sum_{i \in N} t_{i}(R)
$$




\subsection{Proof of Theorem 1}

Throughout this subsection, we assume that $\mathcal{R}$ is rich. Our proof employes many results of Kazumura et al. (2020B). We omit the proofs of such results, and write only the proofs of our new results.

We introduce the concept of favoring preferences. Given $a \in M$, let $\mathcal{R}^{a} \equiv\left\{R_{i} \in \mathcal{R}\right.$ : $\left.\forall b \in M \backslash\{a\},(a, 0) P_{i}(b, 0)\right\}$. By the richness of domains, for each $a \in M, \mathcal{R}^{a} \neq \emptyset$. We say a preference $R_{i} \in \mathcal{R}^{a}$ is $a$-favoring.

Definition 9. Given $(a, t) \in M \times \mathbb{R}_{+}, R_{i}^{\prime} \in \mathcal{R}^{a}$ is $(a, t)$-favoring if for each $b \in M \backslash\{a\}$, $V^{R_{i}^{\prime}}(b ;(a, t))<0$.

Note that for each $R \in \mathcal{R}^{n}$, each $i \in N$ and each $z \in Z(R)$, if $x_{i} \neq 0$, then there is a $z_{i}$-favoring preference $R_{i}^{\prime} \in \mathcal{R}$.

Remark 2. Let $f$ satisfy no subsidy. Let $R \in \mathcal{R}^{n}, i \in N$, and $z \in Z(R)$ be such that $x_{i} \neq 0$ and $R_{i}$ is $z_{i}$-favoring. If $x_{i}(R) \neq x_{i}$, then $z_{i} P_{i} f_{i}(R)$.

Proof. Let $x_{i}(R) \neq x_{i}$. Suppose $f_{i}(R) R_{i} z_{i}$. Then, $t_{i}(R) \leq V^{R_{i}}\left(x_{i}(R) ; z_{i}\right)$. Since $R_{i}$ is $z_{i}$-favoring, $V^{R_{i}}\left(x_{i}(R) ; z_{i}\right)<0$. Thus, $t_{i}(R)<0$, which contradicts no subsidy.

We say a preference $R_{i}$ is $(a, t)^{\varepsilon}$-favoring if at price $p \in \mathbb{R}_{+}^{m}$ such that $p_{a}=t$ and $p_{b}=0$ for each $b \neq a$, agent $i$ demands only object $a$, but when all objects' prices slightly increase, then he depends nothing.

Definition 10. Given $(a, t) \in M \times \mathbb{R}_{+}$and $\varepsilon_{i}>0, R_{i}$ is $(a, t)^{\varepsilon_{i}}$-favoring if $R_{i}$ is $(a, t)$ favoring and

$$
V^{R_{i}}(a ;(0,0))<t+\varepsilon_{i} \text { and } V^{R_{i}}(b ;(0,0))<\varepsilon_{i} \text { for each } b \in M \backslash\{a\} .
$$

Given $a \in M$ and a preference $R_{i} \in \mathcal{R}^{a}$, let $t^{*}\left(R_{i}, a\right) \equiv \min _{b \in M \backslash\{a\}}\left\{V^{R_{i}}(a ;(b, 0))\right\}$.

Remark 3. Let $a \in M$ and $R_{i} \in \mathcal{R}^{a}$. (i) $R_{i}$ is $(a, t)$-favoring preference if and only if $t^{*}\left(R_{i}, a\right)>t$. (ii) For each $b \in L \backslash\{a\}$ and each $t \in\left[0, t^{*}\left(R_{i}, a\right)\right), V^{R_{i}}(b ;(a, t))<0$.

Note that even if all object price is zero except object $a$, agent $i$ with preference $R_{i} \in \mathcal{R}^{a}$ demands only object $a$ if object $a$ 's price is less than $t^{*}\left(R_{i}, a\right)$. Given $a \in M$ and $R_{i} \in \mathcal{R}^{a}, t^{*}\left(R_{i}, a\right)>0$ and we call $t^{*}\left(R_{i}, a\right)$ the supremum $a$-favoring payment for $R_{i}$.

Remark 4. Let $(a, t) \in M \times \mathbb{R}_{+}$and $R_{i} \in \mathcal{R}$ be $(a, t)$-favoring preference. Then, $t^{*}\left(R_{i}, a\right)>t$.

Remark 5. Let $(a, t) \in M \times \mathbb{R}_{+}, \varepsilon>0$ and $R_{i} \in \mathcal{R}$ be $(a, t)^{\varepsilon}$-favoring preference. Then, $0<t^{*}\left(R_{i}, a\right)-t<\varepsilon$. 
Facts 10 and 11 do not depend on the numbers of agents and objects.

Fact 10 (Lemma 2 in Kazumura et al., 2020B). Let $\mathcal{R}$ be rich. Then, for each $(a, t) \in$ $M \times \mathbb{R}_{+}$and each $\varepsilon>0$, there is $R_{i} \in \mathcal{R}$ such that it is $(a, t)^{\varepsilon}$-favoring.

Fact 11 (Lemma 3 in Kazumura et al., 2020B). Let $f$ be desirable and satisfy no subsidy. For each $R \in \mathcal{R}^{n}$, each $i \in N$, and each $t \geq 0$, if there is $j \neq i$ such that $R_{j}$ is $\left(x_{i}(R), t\right)$ favoring, then $t_{i}(R)>t$.

Lemma 1. Let $f$ satisfy individual rationality and strategy-proofness. Let $R \in \mathcal{R}^{n}$ and $z \in Z(R)$. Assume that there is $i \in N$ such that $z_{i} P_{i} f_{i}(R)$. Then, (i) there are $\varepsilon_{i} \in\left(0, V^{R_{i}}\left(x_{i} ; f_{i}(R)\right)-t_{i}\right)$ and $z_{i}^{\varepsilon_{i}}$-favoring preference $R_{i}^{\prime} \in \mathcal{R}$, and (ii) $x_{i}\left(R_{i}^{\prime}, R_{-i}\right) \neq x_{i}$.

Proof. (i) By $z_{i} P_{i} f_{i}(R), t_{i}<V^{R_{i}}\left(x_{i} ; f_{j}(R)\right)$. Thus, there is $\varepsilon_{i} \in\left(0, V^{R_{i}}\left(x_{i} ; f_{i}(R)\right)-t_{i}\right)$. Moreover, by $z_{i} P_{i} f_{i}(R), z \in Z(R)$ and Fact $8, x_{i} \neq 0$, Thus by Fact 10 , there is $z_{i}^{\varepsilon_{i}}$ favoring preference $R_{i}^{\prime} \in \mathcal{R}$.

(ii) Suppose $x_{i}\left(R_{i}^{\prime}, R_{-i}\right)=x_{i}$. Then, by individual rationality, $t_{i}\left(R_{i}^{\prime}\right) \leq V^{R_{i}^{\prime}}\left(x_{i} ;(0,0)\right)$, and by $(\mathrm{i}), V^{R_{i}^{\prime}}\left(x_{i} ;(0,0)\right)<V^{R_{i}}\left(x_{i} ; f_{i}(R)\right)$. Thus, $t_{i}\left(R_{i}^{\prime}\right)<V^{R_{i}}\left(x_{i} ; f_{j}(R)\right)$, and hence $f_{i}\left(R_{i}^{\prime}, R_{-i}\right) P_{i} f_{i}(R)$. This contradicts strategy-proofness. Thus, $x_{i}\left(R_{i}^{\prime}, R_{-i}\right) \neq x_{i}$.

Lemma 2. Let $f$ be desirable and satisfy no subsidy. Let $R \in \mathcal{R}^{n}$ and $z \in Z(R)$. Let $N^{\prime} \subseteq N$ and $R_{N^{\prime}}^{\prime} \in \mathcal{R}^{\left|N^{\prime}\right|}$ be such that for each $i \in N^{\prime}, x_{i} \neq 0$ and $R_{i}^{\prime}$ is $z_{i}$-favoring. Then,

(i) for each $i \in N^{\prime}$, there is $j \in N$ such that $x_{j}\left(R_{N^{\prime}}^{\prime}, R_{-N^{\prime}}\right)=x_{i}$ and

(ii) if $i \neq j$, then $t_{j}\left(R_{N^{\prime}}^{\prime}, R_{-N^{\prime}}\right) \geq t^{*}\left(R_{i}^{\prime}, x_{i}\right)>t_{i}$.

Proof. (i) Let $i \in N^{\prime}$. Then $x_{i} \neq 0$. Suppose that for each $j \in N, x_{j}\left(R_{N^{\prime}}^{\prime}, R_{-N^{\prime}}\right) \neq x_{i}$. Since $x_{i}\left(R_{N^{\prime}}^{\prime}, R_{-N^{\prime}}\right) \neq x_{i}$ and $R_{i}^{\prime}$ is $z_{i}$-favoring, by Remark 2 ,

$$
\left(x_{i}, 0\right) \underset{\text { by } 0 \leq t_{i}}{R_{i}^{\prime}} z_{i} \underset{\text { by Remark } 2}{P_{i}^{\prime}} f_{i}\left(R_{N^{\prime}}^{\prime}, R_{-N^{\prime}}\right),
$$

which contradicts non-wastefulness.

(ii) Let $i \in N^{\prime}$ and $j \in N$ be such that $i \neq j$ and $x_{j}\left(R_{N^{\prime}}^{\prime}, R_{-N^{\prime}}\right)=x_{i}$. Since $R_{i}^{\prime}$ is $z_{i}$-favoring, by Remark 3 (ii), for each $t \in\left[0, t^{*}\left(R_{i}^{\prime}, x_{i}\right)\right), R_{i}^{\prime}$ is $\left(x_{i}, t\right)$-favoring. Thus by Fact $11, t_{j}(R) \geq t^{*}\left(R_{i}^{\prime}, x_{i}\right)$, and by Remark $4, t_{i}<t^{*}\left(R_{i}^{\prime}, x_{i}\right)$. Thus, $t_{j}\left(R_{N^{\prime}}^{\prime}, R_{-N^{\prime}}\right) \geq$ $t^{*}\left(R_{i}^{\prime}, x_{i}\right)>t_{i}$.

Proposition 1. Let $\mathcal{R}$ be rich. Let $f$ be desirable and satisfy no subsidy. For each $R \in \mathcal{R}^{n}$, each $z \in Z^{\min }(R)$ and each $i \in N, f_{i}(R) R_{i} z_{i}$.

Proof. Let $R \in \mathcal{R}^{n}, p=p^{\min }(R)$ and $z \in Z^{\text {min }}(R)$. Let

$$
\underline{p} \equiv \begin{cases}\min \left\{p_{a} \in \mathbb{R}: a \in M \text { and } p_{a}>0\right\} & \text { if } \exists a \in M \text { such that } p_{a}>0 \\ 0 & \text { otherwise }\end{cases}
$$

Suppose that there is $i \in N$ such that $z_{i} P_{i} f_{i}(R)$. Without loss of generality, let $i \equiv 1$. 
Claim. For each $k \geq 0$, there are sets $N(k)$ and $N(k+1)$ of distinct agents such that $N(k+1) \supseteq N(k),|N(k)|=k,|N(k+1)|=k+1$, say $N(k)=\{1,2, \ldots, k\}, N(k+1)=$ $\{1,2, \ldots, k+1\}$, and $\left(\varepsilon_{j}\right)_{j \in N(k+1)} \in \mathbb{R}_{++}^{k+1}, R^{(k)} \equiv\left(R_{N(k)}^{\prime}, R_{-N(k)}\right) \in \mathcal{R}^{n}$ and $R^{(k+1)} \equiv$ $\left(R_{N(k+1)}^{\prime}, R_{-N(k+1)}\right) \in \mathcal{R}^{n}$ such that

(i-a) $z_{k+1} P_{k+1} f_{k+1}\left(R^{(k)}\right)$ and

(i-b) $x_{k+1} \neq 0$,

(ii-a) $\varepsilon_{1}<\min \left(\left\{\underline{p}, V^{R_{1}}\left(x_{1} ; f_{1}(R)\right)-t_{1}\right\} \backslash\{0\}\right)$ and $R_{1}^{\prime}$ is $z_{1}^{\varepsilon_{1}}$-favoring,

(ii-b) for each $j \in N(k+1) \backslash\{1\}, \varepsilon_{j}<\min \left\{t^{*}\left(R_{j-1}^{\prime}, x_{j-1}\right)-t_{j-1}, V^{R_{j}}\left(x_{j} ; f_{j}\left(R^{(j-1)}\right)\right)-t_{j}\right\}$ and $R_{j}^{\prime}$ is $z_{j}^{\varepsilon_{j}}$-favoring,

(ii-c) for each $j \in N(k), \varepsilon_{k+1}<t^{*}\left(R_{j}^{\prime}, x_{j}\right)$,

(iii) $x_{k+1}\left(R^{(k+1)}\right) \neq x_{k+1}$ and $z_{k+1} P_{k+1}^{\prime} f_{k+1}\left(R^{(k+1)}\right)$,

(iv) $x_{k+1}\left(R^{(k+1)}\right) \notin\left\{x_{l}\right\}_{l \in N(k+1)}$, and

(v) there is $j \in N \backslash N(k+1)$ such that $x_{j} \in\left\{x_{l}\right\}_{l \in N(k+1)}$ and $z_{j} P_{j} f_{j}\left(R^{(k+1)}\right)$.

We prove Claim by induction on $k$.

Base Case. Let $k=0$.

(i) By assumption, $z_{1} P_{1} f_{1}(R)$. Thus, (i-a) holds. By (i-a), $z \in Z(R)$ and Fact 8 , $x_{1} \neq 0$. Hence, $(\mathrm{i}-\mathrm{b})$ holds.

(ii) By $\left.z_{1} P_{1} f_{1}(R), t_{1}<V^{R_{1}}\left(x_{1} ; f_{1}(R)\right)\right)$. Thus, there is $\varepsilon_{1}>0$ such that $\varepsilon_{1}<$ $\min \left(\left\{p, V^{R_{1}}\left(x_{1} ; f_{1}(R)\right)-t_{1}\right\} \backslash\{0\}\right)$. By (i-b), $x_{1} \neq 0$. Thus, by Fact 10 , there is $z_{1}^{\varepsilon_{1}}-$ favoring preference $R_{1}^{\prime} \in \mathcal{R}$. Hence, (ii-a) holds. By $k=0$, (ii-b) and (ii-c) hold vacantly.

(iii) By (i-a), (ii-a) and Lemma 1 (ii), $x_{1}\left(R^{(1)}\right) \neq x_{1}$. Thus since $R_{1}^{\prime}$ is $z_{1}^{\varepsilon_{1}}$-favoring, by Remark $2, z_{1} P_{1}^{\prime} f_{1}\left(R^{(1)}\right)$.

(iv) By $k=0$, (iv) directly follows from (iii).

(v) By $x_{1}\left(R^{(1)}\right) \neq x_{1}$ and Lemma $2(\mathrm{i})$, there is $j \in N \backslash\{1\}$ such that $x_{j}\left(R^{(1)}\right)=x_{1}$. Without loss of generality, let $j \equiv 2$. We show that $z_{2} P_{2} f_{2}\left(R^{(1)}\right)$. By Lemma 2 (ii) and $(z, p) \in W(R), t_{2}\left(R^{(1)}\right)>t_{1}=p_{x_{1}}$. Thus,

$$
z_{2} \underset{\text { by }(z, p) \in W(R)}{R_{2}}\left(x_{1}, p_{x_{1}}\right) \underset{\text { by } t_{2}\left(R^{(1)}\right)>p_{x_{1}}}{P_{2}}\left(x_{1}, t_{2}\left(R^{(1)}\right)\right) \underset{\text { by } x_{2}\left(R^{(1)}\right)=x_{1}}{=} f_{2}\left(R^{(1)}\right) .
$$

Thus, $z_{2} P_{2} f_{2}\left(R^{(1)}\right)$.

Inductive Hypothesis. Let $k \geq 1$. There are sets $N(k-1)$ and $N(k)$ of distinct agents such that $N(k) \supseteq N(k-1),|N(k-1)|=k-1,|N(k)|=k$, say $N(k-1)=\{1,2, \ldots, k-1\}$, $N(k)=\{1,2, \ldots, k\}$, and $\left(\varepsilon_{j}\right)_{j \in N(k)} \in \mathbb{R}_{++}^{k}, R^{(k-1)} \equiv\left(R_{N(k-1)}^{\prime}, R_{-N(k-1)}\right) \in \mathcal{R}^{n}$ and $R^{(k)} \equiv\left(R_{N(k)}^{\prime}, R_{-N(k)}\right) \in \mathcal{R}^{n}$ such that

(i-a-k) $z_{k} P_{k} f_{k}\left(R^{(k-1)}\right)$ and

$(\mathrm{i}-\mathrm{b}-\mathrm{k}) x_{k} \neq 0$,

(ii-a-k) $\varepsilon_{1}<\min \left(\left\{\underline{p}, V^{R_{1}}\left(x_{1} ; f_{1}(R)\right)-t_{1}\right\} \backslash\{0\}\right)$ and $R_{1}^{\prime}$ is $z_{1}^{\varepsilon_{1}}$-favoring,

(ii-b-k) for each $j \in N(k), \varepsilon_{j}<\min \left\{t^{*}\left(R_{j-1}^{\prime}, x_{j-1}\right)-t_{j-1}, V^{R_{j}}\left(x_{j} ; f_{j}\left(R^{(j-1)}\right)\right)-t_{j}\right\}$ and $R_{j}^{\prime}$ is $z_{j}^{\varepsilon_{j}}$-favoring, 
(ii-c-k) for each $j \in N(k-1), \varepsilon_{k}<t^{*}\left(R_{j}^{\prime}, x_{j}\right)$,

(iii-k) $x_{k}\left(R^{(k)}\right) \neq x_{k}$ and $z_{k} P_{k}^{\prime} f_{k}\left(R^{(k)}\right)$,

(iv-k) $x_{k}\left(R^{(k)}\right) \notin\left\{x_{l}\right\}_{l \in N(k)}$, and

$(\mathrm{v}-\mathrm{k})$ there is $j \in N \backslash N(k)$ such that $x_{j} \in\left\{x_{l}\right\}_{l \in N(k)}$ and $z_{j} P_{j} f_{j}\left(R^{(k)}\right)$.

\section{Inductive Step.}

(i) By (iv-k), there is $j \in N \backslash N(k)$ such that $z_{j} P_{j} f_{j}\left(R^{(k)}\right)$. Without loss of generality, let $j=k+1$. Then, (i-a) holds. By (i-a), $z \in Z(R)$ and Fact $8, x_{k+1} \neq 0$. Thus, (i-b) holds.

(ii) The hypothesis (ii-a-k) is equivalent to (ii-a).

Next we show (ii-b). By (i-a), $t_{k+1}<V^{R_{k+1}}\left(x_{k+1} ; f_{k+1}\left(R^{(k)}\right)\right)$. By Remark 5 and the hypothesis (ii-b-k), $t^{*}\left(R_{k}^{\prime}, x_{k}\right)-t_{k}>0$. Thus, there is $\varepsilon_{k+1}>0$ such that $\varepsilon_{k+1}<$ $\min \left\{t^{*}\left(R_{k}^{\prime}, x_{k}\right)-t_{k}, V^{R_{k+1}}\left(x_{k+1} ; f_{k+1}\left(R^{(k)}\right)\right)-t_{k+1}\right\}$. By (i-b), $x_{k+1} \neq 0$. Thus, by Fact 10 , there is $z_{k+1}^{\varepsilon_{k+1}}$-favoring preference $R_{k+1}^{\prime} \in \mathcal{R}$. Hence by (ii-b-k), (ii-b) holds.

Note that $\varepsilon_{k+1}<t^{*}\left(R_{k}^{\prime}, x_{k}\right)-t_{k} \leq t^{*}\left(R_{k}^{\prime}, x_{k}\right)$, and so $\varepsilon_{k+1}<t^{*}\left(R_{k}^{\prime}, x_{k}\right)$. By Remark $5, \varepsilon_{k+1}<t^{*}\left(R_{k}^{\prime}, x_{k}\right)-t_{k}<\varepsilon_{k}$, and so by (ii-c-k), for each $j \in N(k) \backslash\{k\}, \varepsilon_{k+1}<\varepsilon_{k}<$ $t^{*}\left(R_{j}^{\prime}, x_{j}\right)$. Thus, for each $j \in N(k), \varepsilon_{k+1}<t^{*}\left(R_{j}^{\prime}, x_{j}\right)$. Hence (ii-c) holds.

(iii) By (i-a), (ii-a) and Lemma 1 (ii), $x_{k+1}\left(R^{(k+1)}\right) \neq x_{k+1}$. Thus since $R_{k+1}^{\prime}$ is $z_{k+1^{-}}$ favoring, by Remark $2, z_{k+1} P_{k+1}^{\prime} f_{k+1}\left(R^{(k+1)}\right)$.

(iv) Suppose that $x_{k+1}\left(R^{(k+1)}\right) \in\left\{x_{l}\right\}_{l \in N(k+1)}$. By (iii), since $x_{k+1}\left(R^{(k+1)}\right) \neq x_{k+1}$, there is $j \in N(k+1) \backslash\{k+1\}$ such that $x_{k+1}\left(R^{(k+1)}\right)=x_{j}$. By $x_{k+1}\left(R^{(k+1)}\right) \neq x_{k+1}$ and (ii-b), $V^{R_{k+1}^{\prime}}\left(x_{k+1}\left(R^{(k+1)}\right) ;(0,0)\right)<\varepsilon_{k+1}$. By individual rationality, $t_{k+1}\left(R^{(k+1)}\right) \leq$ $V^{R_{k+1}^{\prime}}\left(x_{k+1}\left(R^{(k+1)}\right) ;(0,0)\right)$, and so $t_{k+1}\left(R^{(k+1)}\right)<\varepsilon_{k+1}$. By (ii-c), $\varepsilon_{k+1}<t^{*}\left(R_{j}^{\prime}, x_{j}\right)$, and so $t_{k+1}\left(R^{(k+1)}\right)<t^{*}\left(R_{j}^{\prime}, x_{j}\right)$. However, by Lemma 2 (ii), $t_{k+1}\left(R^{(k+1)}\right) \geq t^{*}\left(R_{j}^{\prime}, x_{j}\right)$. This is a contradiction. Thus, $x_{k+1}\left(R^{(k+1)}\right) \notin\left\{x_{l}\right\}_{l \in N(k+1)}$.

(v) By (ii-a), (ii-b) and Lemma 2 (i), for each $i \in N(k+1)$, there is $j \in N$ such that $x_{j}\left(R^{(k+1)}\right)=x_{i}$. By (iv), since $x_{k+1}\left(R^{(k+1)}\right) \notin\left\{x_{l}\right\}_{l \in N(k+1)}$, there is $j \in N \backslash N(k+1)$ such that $x_{j}\left(R^{(k+1)}\right) \in\left\{x_{l}\right\}_{l \in N(k+1)}$.

By Lemma 2 (ii) and $(z, p) \in W(R), t_{j}\left(R^{(k+1)}\right)>t_{j}=p_{x_{j}\left(R^{(k+1)}\right)}$. Thus,

$$
\begin{array}{rrrr}
z_{j} & R_{j} & \left(x_{j}\left(R^{(k+1)}\right), p_{x_{j}\left(R^{(k+1)}\right)}\right) & \text { by }(z, p) \in W(R) \\
& P_{j} & \left(x_{j}\left(R^{(k+1)}\right), t_{j}\left(R^{(k+1)}\right)\right) & \text { by } t_{j}\left(R^{(k+1)}\right)>p_{x_{j}\left(R^{(k+1)}\right)} \\
= & f_{j}\left(R^{(k+1)}\right) . &
\end{array}
$$

Hence $z_{j} P_{j} f_{j}\left(R^{(k+1)}\right)$. The proof of Claim is completed.

By the above Claim, we derive a contradiction. For $k=n-1$, by (i-b), for each $i \in N$, $x_{i} \neq 0$. If $n>m$, it is impossible. Thus, we assume that $n \leq m$.

By (ii-a), (ii-b) and Lemma 2 (i), for each $i \in N$, there is $j \in N$ such that $x_{j}\left(R^{(n)}\right)=x_{i}$. However, by Claim (iv), since $x_{n}\left(R^{(n)}\right) \notin\left\{x_{i}\right\}_{i \in N}$, this is also impossible.

Proof of Theorem 1. By Proposition 1 and Fact 9, a minimum price Walrasian rule is expost revenue optimal in the class of desirable rules satisfying no subsidy. The uniqueness of 
the ex-post revenue optimal rule directly follows from the proof of Theorem 1 in Kazumura et al. (2020B).

\subsection{Proof of Theorem 2}

Throughout this subsection, we assume that $\mathcal{R} \supseteq \mathcal{R}^{++}$and $l \leq 0$ is the associated lower bound of no bankruptcy.

Given $a \in M$ and $\delta>0$, define $\mathcal{R}^{a}(\delta) \equiv\left\{R_{i} \in \mathcal{R}: \forall b \in M \backslash\{a\},(a, 0) P_{i}(b,-\delta)\right\}$. Note that for each $a \in M$ and each $\delta>0$, there is $R_{i} \in \mathcal{R}^{++}$such that $R_{i} \in \mathcal{R}^{a}(\delta)$. Thus, for each $a \in M$ and each $\delta>0, \mathcal{R}^{a}(\delta) \neq \emptyset$.

Definition 11. Given $(a, t) \in M \times \mathbb{R}_{+}$and $\delta>0, R_{i}^{\prime} \in \mathcal{R}^{a}(\delta)$ is $(a, t)$-favoring with subsidy $\delta$ if for each $b \in M \backslash\{a\}, V^{R_{i}^{\prime}}(b ;(a, t))<-\delta$.

Note that for each $R \in \mathcal{R}^{n}$, each $z \in Z(R)$ and each $\delta>0$, if $x_{i} \neq 0$, by $\mathcal{R} \supseteq \mathcal{R}^{++}$, there is a $z_{i}$-favoring preference $R_{i}^{\prime}$ with subsidy $\delta$.

Given $a \in M, \delta>0$ and $R_{i} \in \mathcal{R}^{a}(\delta)$, let $t^{*}\left(R_{i}, a, \delta\right) \equiv \min _{b \in M \backslash\{a\}}\left\{V^{R_{i}}(a ;(b,-\delta))\right\}$.

Remark 6. Let $a \in M, \delta>0$ and $R_{i} \in \mathcal{R}^{a}(\delta)$. (i) $R_{i}$ is $(a, t)$-favoring with subsidy $\delta$ if and only if $t^{*}\left(R_{i}, a, \delta\right)>t$. (ii) For each $b \in L \backslash\{a\}$ and each $t \in\left[0, t^{*}\left(R_{i}, a, \delta\right)\right)$, $V^{R_{i}}(b ;(a, t))<-\delta$.

For each $a \in M$, each $\delta>0$ and each $R_{i} \in \mathcal{R}^{a}(\delta), t^{*}\left(R_{i}, a, \delta\right)>0$.

Given $(a, t) \in M \times \mathbb{R}_{+}, \varepsilon>0$ and $\delta>0$, let $\mathcal{R}((a, t), \varepsilon, \delta)$ be the set of $(a, t)^{\varepsilon}$-favoring preferences in $\mathcal{R}^{a}(\delta)$.

Fact 12. For each $(a, t) \in M \times \mathbb{R}_{+}$, each $\varepsilon>0$ and each $\delta>0, \mathcal{R}^{++} \cap \mathcal{R}((a, t), \varepsilon, \delta) \neq \emptyset$.

Remark 7. Let $(a, t) \in M \times \mathbb{R}_{+}, \varepsilon>0$ and $R_{i}^{\prime} \in \mathcal{R}^{++} \cap \mathcal{R}((a, t), \varepsilon, \delta)$. Then, (i) $t^{*}\left(R_{i}, a, \delta\right)>t$ and (ii) $0<t^{*}\left(R_{i}, a, \delta\right)-t<\varepsilon$.

Given $R \in \mathcal{R}^{n}$ and $l \leq 0$, let $\delta(R, l) \equiv n\left(\max _{k \in N} \max _{b \in M} V^{R_{k}}(b ;(0,0))\right)-l$.

Fact 13 (Lemma 4 in Kazumura et al., 2020B). Let $f$ be desirable and satisfy no bankruptcy. For each $R \in \mathcal{R}^{n}$, each $i \in N$, and each $(a, t) \in M \times \mathbb{R}_{+}$, if there is $j \neq i$ such that for each $a \in L \backslash\left\{x_{i}(R)\right\}, V^{R_{j}}\left(a ; f_{i}(R)\right)<-\delta(R, l)$, then $t_{i}(R)>t$.

Lemma 3. Let $f$ satisfy individual rationality and no bankruptcy. For each $R \in \mathcal{R}^{n}$ and $i \in N, t_{i}(R) \geq-\delta(R, l)$. 
Proof. Suppose that there is $i \in N$ such that $t_{i}(R)<-\delta(R, l)$. Note that by individual rationality, for each $j \in N \backslash\{i\}, t_{j}(R) \leq \max _{b \in M} V^{R_{j}}(b ;(0,0))$. Thus,

$$
\begin{aligned}
\sum_{k \in N} t_{k}(R) & \leq \sum_{j \in N \backslash\{i\}} \max _{b \in M} V^{R_{j}}(b ;(0,0))-\delta(R, l) \\
& \leq(n-1) \max _{k \in N} \max _{b \in M} V^{R_{k}}(b ;(0,0))-\delta(R, l) \\
& =(n-1) \max _{k \in N} \max _{b \in M} V^{R_{k}}(b ;(0,0))-n\left(\max _{k \in N} \max _{b \in M} V^{R_{k}}(b ;(0,0))\right)+l \\
& <l
\end{aligned}
$$

which contradicts no bankruptcy.

Remark 8. Let $f$ satisfy individual rationality and no bankruptcy. Let $R \in \mathcal{R}^{n}, i \in N$, and $z \in Z(R)$ be such that $x_{i} \neq 0$ and $R_{i} \in \mathcal{R}^{++} \cap \mathcal{R}\left(z_{i}, \varepsilon, \delta(R, l)\right)$. If $x_{i}(R) \neq x_{i}$, then $z_{i} P_{i} f_{i}(R)$.

Proof. Let $x_{i}(R) \neq x_{i}$. Suppose $f_{i}(R) R_{i} z_{i}$. Then, $t_{i}(R) \leq V^{R_{i}}\left(x_{i}(R) ; z_{i}\right)$. Since $R_{i} \in$ $\mathcal{R}\left(z_{i}, \varepsilon, \delta(R, l)\right), V^{R_{i}}\left(x_{i}(R) ; z_{i}\right)<-\delta(R, l)$. Thus, $t_{i}(R)<-\delta(R, l)$, which contradicts Lemma 3.

Lemma 4. Let $f$ satisfy individual rationality and strategy-proofness. Let $R \in \mathcal{R}^{n}$ and $z \in Z(R)$. Assume that there is $i \in N$ such that $z_{i} P_{i} f_{i}(R)$. Then, (i) there are $\varepsilon_{i}>0$ and $R_{i}^{\prime} \in \mathcal{R}$ such that $\varepsilon_{i}<V^{R_{i}}\left(x_{i} ; f_{i}(R)\right)-t_{i}$ and $R_{i}^{\prime} \in \mathcal{R}^{++} \cap \mathcal{R}\left(z_{i}, \varepsilon_{i}, \delta(R, l)\right)$. (ii) $x_{i}\left(R_{i}^{\prime}, R_{-i}\right) \neq x_{i}$.

Proof. (i) By $z_{i} P_{i} f_{i}(R), t_{i}<V^{R_{i}}\left(x_{i} ; f_{j}(R)\right)$. Thus, there is $\varepsilon_{i} \in\left(0, V^{R_{i}}\left(x_{i} ; f_{i}(R)\right)-t_{i}\right)$. Moreover, by $z_{i} P_{i} f_{i}(R) z \in Z(R)$ and Fact $8, x_{i} \neq 0$. Thus, by Fact 12 , there is $R_{i}^{\prime} \in \mathcal{R}^{++} \cap \mathcal{R}\left(z_{i}, \varepsilon, \delta(R, l)\right)$

(ii) Directly follows from the proof of Lemma 1 (ii).

Lemma 5. Let $f$ be desirable and satisfy no bankruptcy. Let $R \in \mathcal{R}^{n}$ and $z \in Z(R)$. Let $N^{\prime} \subseteq N$ and $R_{N^{\prime}}^{\prime} \in \mathcal{R}^{\left|N^{\prime}\right|}$ be such that for each $i \in N^{\prime}, x_{i} \neq 0$ and $R_{i}^{\prime} \in \mathcal{R}^{++} \cap$ $\mathcal{R}\left(z_{i}, \varepsilon, \delta(R, l)\right)$. Then,

(i) for each $i \in N^{\prime}$, there is $j \in N$ such that $x_{j}\left(R_{N^{\prime}}^{\prime}, R_{-N^{\prime}}\right)=x_{i}$ and

(ii) if $i \neq j$, then $t_{j}\left(R_{N^{\prime}}^{\prime}, R_{-N^{\prime}}\right) \geq t^{*}\left(R_{i}^{\prime}, x_{i}, \delta(R, l)\right)>t_{i}$

Proof. (i) Let $i \in N^{\prime}$. Suppose that for each $j \in N, x_{j}\left(R_{N^{\prime}}^{\prime}, R_{-N^{\prime}}\right) \neq x_{i}$. Since $x_{i}\left(R_{N^{\prime}}^{\prime}, R_{-N^{\prime}}\right) \neq x_{i}$ and $R_{i}^{\prime}$ is $z_{i}$-favoring, by Remark 8 ,

$$
\left(x_{i}, 0\right) \underset{\text { by } 0 \leq t_{i}}{R_{i}^{\prime}} z_{i} \underset{\text { by Remark } 8}{P_{i}^{\prime}} f_{i}\left(R_{N^{\prime}}^{\prime}, R_{-N^{\prime}}\right)
$$

which contradicts non-wastefulness.

(ii) Let $i \in N^{\prime}$ and $j \in N$ be such that $i \neq j$ and $x_{j}\left(R_{N^{\prime}}^{\prime}, R_{-N^{\prime}}\right)=x_{i}$. Since $R_{i}^{\prime}$ is $z_{i}$-favoring, by Remark 6 (ii), for each $t \in\left[0, t^{*}\left(R_{i}^{\prime}, x_{i}\right)\right), R_{i}^{\prime}$ is $\left(x_{i}, t\right)$-favoring. Thus by Fact $13, t_{j}(R) \geq t^{*}\left(R_{i}^{\prime}, x_{i}, \delta(R, l)\right)$. By Remark 7 (i), $t_{i}<t^{*}\left(R_{i}^{\prime}, x_{i}, \delta(R, l)\right)$. Thus, $t_{j}\left(R_{N^{\prime}}^{\prime}, R_{-N^{\prime}}\right) \geq t^{*}\left(R_{i}^{\prime}, x_{i}, \delta(R, l)\right)>t_{i}$. 
Proposition 2. Let $\mathcal{R} \supseteq \mathcal{R}^{++}$. Let $f$ be desirable and satisfy no bankruptcy. For each $R \in \mathcal{R}^{n}$, each $z \in Z^{\min }(R)$ and each $i \in N, f_{i}(R) R_{i} z_{i}$.

Proof. Let $R \in \mathcal{R}^{n}, p=p^{\min }(R)$ and $z \in Z^{\text {min }}(R)$. Let

$$
\underline{p} \equiv \begin{cases}\min \left\{p_{a} \in \mathbb{R}: a \in M \text { and } p_{a}>0\right\} & \text { if } \exists a \in M \text { such that } p_{a}>0 \\ 0 & \text { otherwise }\end{cases}
$$

Suppose that there is $i \in N$ such that $z_{i} P_{i} f_{i}(R)$. Without loss of generality, let $i \equiv 1$.

Claim. For each $k \geq 0$, there are sets $N(k)$ and $N(k+1)$ of distinct agents such that $N(k+1) \supseteq N(k),|N(k)|=k,|N(k+1)|=k+1$, say $N(k)=\{1,2, \ldots, k\}, N(k+1)=$ $\{1,2, \ldots, k+1\}$, and $\left(\varepsilon_{j}\right)_{j \in N(k+1)} \in \mathbb{R}_{++}^{k+1}, R^{(k)} \equiv\left(R_{N(k)}^{\prime}, R_{-N(k)}\right) \in \mathcal{R}^{n}$ and $R^{(k+1)} \equiv$ $\left(R_{N(k+1)}^{\prime}, R_{-N(k+1)}\right) \in \mathcal{R}^{n}$ such that

(i-a) $z_{k+1} P_{k+1} f_{k+1}\left(R^{(k)}\right)$ and

(i-b) $x_{k+1} \neq 0$,

(ii-a) $\varepsilon_{1}<\min \left(\left\{\underline{p}, t^{*}\left(R_{1}^{\prime}, x_{1}, \delta(R, l)\right)-t_{1}\right\} \backslash\{0\}\right)$ and $R_{1}^{\prime} \in \mathcal{R}^{++} \cap \mathcal{R}\left(z_{1}, \varepsilon_{1}, \delta(R, l)\right)$,

(ii-b) for each $j \in N(k+1) \backslash\{1\}, \varepsilon_{j}<\min \left\{t^{*}\left(R_{j-1}^{\prime}, x_{j-1}, \delta(R, l)\right)-t_{j-1}, V^{R_{j}}\left(x_{j} ; f_{j}\left(R^{(j-1)}\right)\right)-\right.$ $\left.t_{j}\right\}$ and $R_{j}^{\prime} \in \mathcal{R}^{++} \cap \mathcal{R}\left(z_{j}, \varepsilon_{j}, \delta(R, l)\right)$,

(ii-c) for each $j \in N(k), \varepsilon_{k+1}<t^{*}\left(R_{j}^{\prime}, x_{j}, \delta(R, l)\right)$,

(iii) $x_{k+1}\left(R^{(k+1)}\right) \neq x_{k+1}$ and $z_{k+1} P_{k+1}^{\prime} f_{k+1}\left(R^{(k+1)}\right)$,

(iv) $x_{k+1}\left(R^{(k+1)}\right) \notin\left\{x_{l}\right\}_{l \in N(k+1)}$, and

(v) there is $j \in N \backslash N(k+1)$ such that $x_{j} \in\left\{x_{l}\right\}_{l \in N(k+1)}$ and $z_{j} P_{j} f_{j}\left(R^{(k+1)}\right)$

To replace Lemma 1 with Lemma 4, Lemma 2 with Lemma 5, Remark 5 with Remark 7, and Fact 10 with Fact 12, by the same logic in Proposition 1, we can prove the above claim.

Proof of Theorem 2. The same logic in Theorem 1.

\section{Concluding remarks}

By extending the results of Kazumura et al. (2020B), we showed that for an arbitrary numbers of agents and objects, the minimum price Walrasian rule is the unique ex-post revenue maximizing rule on rich domains among desirable rules, and that no subsidy in this result can be replaced by no bankruptcy on the positive income effect domain. There is the literature on auction with non-quasi-linear preferences. We conclude by referring them and mentioning relating research in future.

Most of the literature on auction with non-quasi-linear preferences focus on rather efficiency than revenue maximization. Saitoh and Serizawa (2008) and Sakai (2008) show that in the cases of homogeneous objects and unit-demand preferences, the generalized 
Vickrey rule is the only rule satisfying strategy-proofness, efficiency, individual rationality, and no subsidy. Morimoto and Serizawa (2015) extend these results to the case of heterogeneous objects by maintaining unit-demand preferences, and show that the minimum price Walrasian rule is the only rule satisfying the same four properties on classical domain. These works assume that the number of agents is greater than objects. Thus, it is an open question whether these results hold for an arbitrary numbers of agents and objects.

Zhou and Serizawa (2018) also maintain unit-demand preferences, but study the special class of preferences, the common-tiered domains. It says that objects are partitioned into several tiers, and if objects are equally priced, agents prefer an object in the higher tier to one in the lower. They show that the minimum price Walrasian rule is the only rule satisfying same four properties on the common-tiered domains. It is an open question whether their results also hold on the common-tiered domains for an arbitrary numbers of agents and objects.

There is also the literature on auction with non-quasi-linear preferences admitting multi-demand in various settings. Kazumura and Serizawa (2016) study classes of preferences that include unit-demand preferences and additionally includes at least one multidemand preference, and show that no rule satisfies the four properties on such a domain. Malik and Mishra (2019) study the special classes of preferences, "dichotomous" domains. A preference is dichotomous if there is a set of objects such that the valuations of its supersets are constant and the valuations of other sets are zero. A dichotomous domain includes all such dichotomous preferences for a given set of objects. They show that no rule satisfies the four properties on a dichotomous domain, but that the generalized Vickrey rule is the only rule satisfying the four properties on a class of dichotomous preferences exhibiting positive income effects.

Baisa (2020) assumes that objects are homogeneous and shows that on the class of preferences exhibiting decreasing marginal valuations, positive income effect, and singlecrossing property, if the preferences are parametrized by one dimensional types, there is a rule satisfying the above four properties, but that if types are multi-dimensional, no rule satisfies these properties. Shinozaki et al. (2020) also assume the homogeneity of objects, and show that on the class of preferences includes sufficiently various preferences exhibiting non-decreasing marginal valuations (minimal richness), the generalized Vickrey rule is the only rule satisfying the four properties, but that no rule satisfies these properties on the class of preferences that additionally includes at least one preference exhibiting decreasing marginal valuations.

These different results in various settings of multi-demand suggest that analyzing revenue maximization rules in multi-demand settings would be technically challenging. However, such research is important in practical applications. Recently, Kazumura et al. (2020A) develop methods to analyze strategy-proof rules in general settings including multi-demand cases. We believe that such methods would be useful to analyze revenue maximization rules in multi-demand settings. 


\section{References}

[1] Alkan, A. and D. Gale (1990), The core of the matching game, Games and Economic Behavior, 2, 203-212.

[2] Baisa, B. (2020), Efficient multi-unit auctions for normal goods, Theoretical Economics, 15, 361-423.

[3] Demange, G. and D. Gale (1985), The strategy structure of two-sided matching markets, Econometrica, 53, 873-888.

[4] Kazumura, T., D. Mishra and S. Serizawa (2020A), Mechanism design without quasilinearity, Theoretical Economics, 15, 511-544

[5] Kazumura, T., D. Mishra and S. Serizawa (2020B), Strategy-proof multi-object mechanism design: Ex-post revenue maximization with non-quasilinear preferences, Journal of Economic Theory, 188, 1-30.

[6] Kazumura, T. and S. Serizawa (2016), Efficiency and strategy-proofness in object assignment problems with multi-demand preferences, Social Choice and Welfare, 47, 633-663.

[7] Malik, M. and D. Mishra (2020), Pareto efficient combinatorial auctions: Dichotomous preferences without quasilineality, Working Paper.

[8] Morimoto, S. and S. Serizawa, (2015), Strategy-proofness and efficiency with nonquasi-linear preferences: A characterization of minimum price Walrasian rule, Theoretical Economics, 10, 445-487.

[9] Saitoh, H. and S. Serizawa (2008), Vickrey allocation rule with income effect, Economic Theory, 35, 391-401.

[10] Sakai, T. (2008), Second price auctions on general preference domains: Two characterization, Economic Theory, 37, 347-356.

[11] Shinozaki, H., T. Kazumura and S. Serizawa (2020), Efficient and strategy-proof multi-unit object allocation with money: (Non)decreasing marginal valuations without quasi-linearity, Working Paper.

[12] Zhou, Y. and S. Serizawa (2018), Strategy-proofness and efficiency for non-quasilinear and common-tiered-object preferences: Characterization of minimum price rule, Game and Economic Behavior, 109, 327-363. 